\title{
Voting Systems That Combine Approval and Preference
}

\author{
Steven J. Brams*, M. Remzi Sanver ${ }^{\dagger}$
}

\begin{abstract}
Information on the rankings and information on the approval of candidates in an election, though related, are fundamentally different—one cannot be derived from the other. Both kinds of information are important in the determination of social choices. We propose a way of combining them in two hybrid voting systems, preference approval voting (PAV) and fallback voting (FV), that satisfy several desirable properties, including monotonicity. Both systems may give different winners from standard ranking and nonranking voting systems. PAV, especially, encourages candidates to take coherent majoritarian positions, but it is more information-demanding than FV. PAV and FV are manipulable through voters' contracting or expanding their approval sets, but a 3-candidate dynamic poll model suggests that Condorcet winners, and candidates ranked first or second by the most voters if there is no Condorcet winner, will be favored, though not necessarily in equilibrium.
\end{abstract}

Key words : Approval voting; Ranking systems; Condorcet winner; Manipula- bility; Monotonicity

\section{Introduction}

Social choice theory, while postulating that voters have preferences over candidates, does not ask them to stipulate where, in their preference rankings, they would draw the line between acceptable and unacceptable candidates. Approval voting (AV) does ask voters to draw such a line, but it ignores rankings above and below this line.

\footnotetext{
${ }^{*}$ Department of Politics, New York University, New York, NY 10003, USA, steven.brams@nyu.edu

${ }^{\dagger}$ Department of Economics, Istanbul Bilgi University, 80310, Kustepe, Istanbul, TURKEY, sanver@bilgi.edu.tr
} 
Rankings and approval, though related, are fundamentally different kinds of information - one cannot be derived from the other. Both kinds of information are important in the determination of social choices. We propose a way of combining them in two hybrid voting systems, preference approval voting (PAV) and fallback voting $(\mathrm{FV})$, that have several desirable properties.

Approving of a subset of candidates is generally not difficult, whereas ranking all candidates on a ballot, especially if the list is long, may be arduous. PAV asks for both kinds of information, whereas FV asks voters to rank only those candidates they approve of, making it simpler than systems that elicit complete rankings.

We describe, analyze, and compare each of these systems in tandem. In section 2 we give definitions and assumptions. In section 3, we describe PAV and analyze which candidates can and cannot win under this system. Although a PAV winner may not be a Condorcet winner or AV winner, PAV satisfies what we call the strongest-majority principle for voters. More specifically, if a majority-approved candidate is preferred by a majority to the AV winner and other majority-approved candidates, PAV "corrects" the $\mathrm{AV}$ result by electing the majority-preferred candidate.

A majority-preferred candidate is likely to have a more coherent point of view than an AV winner, who may be the most popular candidate because he or she is bland or inoffensive - a kind of lowest common denominator who tries to appease everybody. Sometimes not choosing such a candidate when two or more candidates receive majority approval makes PAV coherence-inducing for candidates by giving an advantage to candidates who are principled but, nevertheless, command broad support.

In section 4 we describe FV and compare its properties with those of PAV. Like PAV, FV tends to help those candidates who are relatively highly ranked by a majority of voters. Both systems may give different winners from nonranking systems (e.g., plurality voting and $\mathrm{AV}$ ), ranking systems (e.g., the Borda count and single transferable vote, or STV), and each other.

In section 5 we show that PAV and FV are monotonic in two different senses: Voters, by either approving of a candidate or raising him or her in their rankings, can never hurt and may help this candidate get elected. The latter property (rank-monotonicity) is not satisfied by a number of ranking systems, including STV, whereas the former property (approval-monotonicity) is satisfied by AV.

Like all voting systems, PAV and FV are manipulable. In section 6 we show that voters may induce preferred outcomes either by contracting or by expanding their approval sets. Because each voting system may give outcomes in equilibrium when the other does not, neither system is inherently more stable than the other.

In section 7 we develop a dynamic model of voter responses to polls in 3-candidate elections, wherein voter preferences are either single-peaked or cyclic. If voters respond 
to successive polls by adjusting their approval strategies to try to prevent their worst choices from winning, they elect the Condorcet winner if their preferences are singlepeaked. If their preferences are cyclical, the candidate ranked first or second by the most voters wins after voters respond to several polls. These outcomes are in equilibrium under both PAV and FV, except, surprisingly, in some instances when voter preferences are single-peaked.

We conclude in section 8 that PAV, and to a less extent FV, subtlely interweave two different kinds of information: Approval information determines those candidates who are sufficiently popular to be serious contenders if not outright winners; ranking information enables voters to refine the set of potential winners if more than one candidate receives majority approval.

Together, these two kinds of information facilitate the election of majoritarian candidates with coherent positions. But more than abetting their election, PAV and FV may well have a salutary impact on which candidates choose to run-and how they choose to campaign - encouraging the entry of candidates who appeal to a broad segment of the electorate but do not promise them the moon.

\section{Definitions and Assumptions}

Consider a set of voters choosing among a set of candidates. We denote individual candidates by small letters $a, b, c, \ldots$.

We assume that voters strictly rank the candidates from best to worst, so there is no indifference. Thus, for any candidates $a$ and $b$, either $a$ is preferred to $b$ or $b$ is preferred to $a$. This assumption simplifies the subsequent analysis but does not in any significant way affect our results, which can readily be extended to the case of nonstrict preferences.

We assume that rankings are transitive, so that for any candidates $a, b$ and $c, a$ is preferred to $c$ whenever $a$ is preferred to $b$ and $b$ is preferred to $c$. In addition, we assume that a voter evaluates each candidate as either acceptable or unacceptable, which we will refer to as approved and disapproved candidates.

The preference-approval of voters is based on both their rankings and their approval of candidates. Although different, these two types of information exhibit the following consistency: Given two candidates $a$ and $b$, if $a$ is approved and $b$ is disapproved, then $a$ is ranked above $b$.

We represent a voter's preference-approval by an ordering of candidates from left to right and a vertical bar, to the left of which candidates are approved and to the right of which candidates are disapproved. For example,

$a b \mid c d$ 
indicates that the voter's two top-ranked candidates, $a$ and $b$, are approved, and the voter's two bottom-ranked candidates, $c$ and $d$, are disapproved.

At one extreme, a voter may approve of all candidates, and at the other extreme of no candidates. As we discuss in section 6, these extreme strategies are dominated strategies in a voting game in which voters have strict preferences, but these strategies are not illegal, as such, under PAV of FV.

Some voters will approve of a single favorite candidate, and some will approve of all except a worst choice. Many voters, however, are likely to select some middle ground, approving of two or three candidates in, say, a field of five (for empirical data on this question under AV, see Brams and Fishburn, 2005).

A preference-approval profile is a list of preference-approvals of all voters. A socialchoice rule, as we use the term here, aggregates preference-approval profiles into social choices. Thereby our framework generalizes the standard social-choice model-wherein a voter is characterized simply by his or her ranking of candidates - to one that adds a line in the ranking separating the voter's approvals from disapprovals.

In subsequent sections, we will use a number of examples to illustrate results as well as prove some propositions. Voters who have the same ranking of candidates will be put into classes, distinguished by Roman numerals $I, I I, I I I, \ldots$.. For simplicity, we assume in the examples that all voters in a class draw the line separating approvals and disapprovals at the same point in their rankings, but none of our results depends on this assumption.

To describe PAV in the next section, we need two definitions. A Condorcet winner is a candidate who is preferred by a majority to every other candidate in pairwise comparisons. A cycle among 3 or more candidates $a, b, c, \ldots$ occurs if $a>b>c>\ldots>a$, where " $>$ " indicates "is preferred by a majority to." The majority preference relation between any two candidates may lead to a tie if and only if there is an even number of voters, which we assume is broken by random tie-breaking.

\section{Preference Approval Voting (PAV)}

The winner under PAV is determined by two rules, the second comprising two cases:

1. If no candidate, or exactly one candidate, receives a majority of approval votes, then the PAV winner is the $\mathrm{AV}$ winner-that is, the candidate who receives the most approval votes.

2. If two or more candidates receive a majority of approval votes, then 
(i) If one of these candidates is preferred by a majority to every other majorityapproved candidate, then he or she is the PAV winner-even if not the AV or Condorcet winner.

(ii) If there is not one majority-preferred candidate because of a cycle among the majority-approved candidates, then the $\mathrm{AV}$ winner among them is the PAV winner-even if not the $\mathrm{AV}$ or Condorcet winner.

It is rule 2 that distinguishes PAV from $\mathrm{AV}$. It allows for the election of candidates who are not the most approved and, therefore, not AV winners. As we will see, a PAV winner may in fact be the least-approved candidate in a race.

Compared with preference-based voting systems, PAV is somewhat more demanding in the information that it requires of voters. Besides ranking candidates, voters must indicate where they draw the line between acceptable and unacceptable candidates, which is an issue we will return to when we compare the complexity of PAV and FV.

In the remainder of this section, we show what kinds of candidates PAV may and may not elect:

Proposition 1 A Condorcet winner may not be a PAV winner under rule 1, rule 2(i), and rule 2(ii).

Proof Rule 1. Consider the following 3-voter, 3-candidate example, in which the voters divide into three preference classes:

\section{Example 1}

- $I .1$ voter: $a b \mid c$

- II. 1 voter: $b \mid a c$

- III. 1 voter: $c \mid a b$

Candidate $b$ is the $\mathrm{AV}$ winner, approved of by 2 of the 3 voters, whereas candidates $a$ and $c$ are approved of by only 1 voter each. Because candidate $b$ is the only candidate approved of by a majority, $b$ is the PAV winner under rule 1 . But it is candidate $a$, who is preferred to candidates $b$ and $c$ by majorities of 2 votes to 1 , that is the Condorcet winner.

Rule 2(i). Consider the following 3-voter, 4-candidate example:

\section{Example 2}

- $I .1$ voter: $a b c \mid d$ 
- $I I .1$ voter: $b c \mid a d$

- III. 1 voter: $d \mid a c b$

Candidates $b$ and $c$ tie for $\mathrm{AV}$ winner with majorities of 2 votes each. Because candidate $b$ is preferred to candidate $c$ by 2 votes to $1, b$ is the PAV winner under rule 2(i). But it is candidate $a$, who is preferred to candidates $b, c$, and $d$ by majorities of 2 votes to 1 (but who is not majority-approved), that is the Condorcet winner.

Rule 2(ii). Consider the following 5-voter, 5-candidate example:

\section{Example 3}

- I. 1 voter: $d a b c \mid e$

- $I I .1$ voter: $d b c a \mid e$

- III. 1 voter: $e \mid d c a b$

- $I V .1$ voter: $a b c \mid d e$

- $V .1$ voter: $c \mid b$ a de

Candidates $a$ (3 votes), $b$ ( 3 votes), and $c$ ( 4 votes) are all majority-approved and in a cycle as well: $a>b>c>a$. Because the Condorcet winner, candidate $d$ (2 votes), is not majority-approved, he or she cannot be the PAV winner. Instead, the most approved candidate in the cycle, $c$, is the PAV winner. Q.E.D.

Not only may PAV fail to elect Condorcet winners when they exist, but it may also fail to elect unanimously approved candidates.

Proposition 2 A unanimously approved $A V$ winner may not be a PAV winner under either rule 2 (i) or rule 2 (ii).

Proof Rule 2(i). Consider the following 3-voter, 3-candidate example:

\section{Example 4}

- $I .2$ voters: $a b \mid c$

- II. 1 voter: $b c \mid a$ 
Candidate $b$ is approved of by all 3 voters, whereas candidate $a$ is approved of by 2 voters and candidate $c$ by 1 voter. Nevertheless, candidate $a$ is the PAV winner, because under rule 2(i) he or she is preferred by 2 votes to 1 to the other majority-approved candidate, $b$.

Rule 2(ii). Consider the following example 8-voter, 4-candidate example:

\section{Example 5}

- $I .3$ voters: $a b c \mid d$

- $I I .3$ voters: $d a c \mid b$

- III. 2 voters: $b d c \mid a$

Candidate $c$ is approved of by all 8 voters, whereas candidates $a, b$, and $d$ are approved of by majorities of either 5 or 6 voters. The latter three candidates are in a top cycle in which $a>b>d>a$; all are preferred by majorities to candidate $c$, the AV winner. But because candidate $a$ receives more approvals (6) than candidates $b$ and $d$ (5 each), candidate $a$ is the PAV winner under rule 2(ii). Q.E.D.

Proposition 2 shows how a unanimously approved AV winner may be displaced by a less approved majority winner under PAV. In fact, the conflict between AV and PAV winners may be even more extreme.

Proposition 3 A least-approved candidate may be a PAV winner under rule 2(i).

Proof Consider the following 7-voter, 4-candidate example:

\section{Example 6}

- $I .2$ voters: $a c b \mid d$

- II. 2 voters: $a c d \mid b$

- III. 3 voters: $b c d \mid a$

Candidate $c$ is approved of by all 7 voters, candidates $b$ and $d$ by 5 voters each, and candidate $a$ by 4 voters. While all candidates receive majority approval, candidate $a$ is the PAV winner, because he or she is preferred by a majority (class $I I$ and $I I I$ voters) to the $\mathrm{AV}$ winner (candidate $c$ ), as well as candidates $b$ and $d$, under rule 2(i) Q.E.D.

When the PAV winner and the AV winner differ, as in Example 6, the PAV winner is arguably the more coherent majority choice. Two of the three classes of voters rank 
candidate $a$ as their top choice in Example 6, whereas candidate $c$, the AV winner, is not the top choice of any class of voters.

Finally, we show that PAV may give winners different from the two-best known ranking systems (for more information on these and other voting systems, see Brams and Fishburn, 2002).

Proposition 4 A PAV winner may be different from winners under the Borda count and single transferable vote (STV).

\section{Proof}

If there are $n$ candidates, the Borda count assigns $n-1$ points to the first choice of a voter, $n-2$ points to the second choice, . . ., 0 points to the last choice; the candidate with the most points wins. In Example 6, candidate $c$ wins with 14 points (2 points each from all 7 voters), whereas the PAV winner, candidate $a$, receives 12 points ( 3 points each from 4 voters and 0 points from 3 voters).

Under STV, only first-place votes are counted initially. In Example 5, candidates $a, d$, and $b$ receive 3, 3, and 2 votes, respectively, from the voters who rank them first. Because candidate $b$ receives the fewest votes, the votes or his or her supporters are transferred to their second choice, candidate $d$, giving $d$ a total of 5 votes, which is a majority and makes candidate $d$ the winner. By contrast, candidate $a$ is the PAV winner. Q.E.D.

In summary, we have shown that PAV may not elect Condorcet winners, or winners under AV, the Borda count, or STV. Nevertheless, PAV winners are strong contenders on grounds of both approval and preference, which we will say more about later.

We turn next to a voting system that is less information-demanding than PAV, requiring voters to rank only those candidates of whom they approve. It shares some properties of PAV but by no means all.

\section{Fallback Voting (FV)}

Fallback voting (FV) proceeds as follows:

1. Voters indicate all candidates of whom they approve, who may range from no candidate (which a voter does by abstaining from voting) to all candidates. Voters rank only those candidates of whom they approve.

2. The highest-ranked candidate of all voters is considered. If a majority of voters agree on one highest-ranked candidate, this candidate is the FV winner. The procedure stops, and we call this candidate a level 1 winner. 
3. If there is no level 1 winner, the next-highest ranked candidate of all voters is considered. If a majority of voters agree on one candidate as either their highest or their next-highest ranked candidate, this candidate is the FV winner. If more than one candidate receives majority approval, then the candidate with the largest majority is the FV winner. The procedure stops, and we call this candidate a level 2 winner.

4. If there is no level 2 winner, the voters descend —one level at a time-to lower and lower ranks of approved candidates, stopping when, for the first time, one or more candidates are approved of by a majority of voters, or no more candidates are ranked. If exactly one candidate receives majority approval, this candidate is the FV winner. If more than one candidate receives majority approval, then the candidate with the largest majority is the FV winner. If the descent reaches the lowest rank of all voters and no candidate is approved of by a majority of voters, the candidate with the most approval is the FV winner.

The appellation "fallback" comes from the fact that FV successively falls back on lower-ranked approved candidates if no higher-ranked approved candidate receives majority approval. This nomenclature was first used in Brams and Kilgour (2001), but it was applied to bargaining rather than voting, in which the decision rule was assumed to be unanimity (the assent of all parties was necessary) rather than a simple majority.

Brams and Kilgour (2001), in what they called "fallback bargaining with impasse," did not require that the bargainers rank all alternatives. Rather, the bargainers ranked only those they considered better than "impasse," because impasse was preferable to any alternative ranked lower. Bargainers not ranking alternatives below impasse are analogous to voters not approving of candidates below a certain level, whom they do not rank.

Like FV, the "majoritarian compromise" proposed by Sertel and his colleagues (Sertel and Yilmaz, 1999; Sertel and Sanver, 1999; Hurwicz and Sertel, 1999) elects the first candidate approved of by a majority in the descent process. However, voters are assumed to rank all candidates- they do not stop their ranking at some point at which they consider candidates they rank lower unacceptable.

Bucklin assumed, as we do with FV, that if a voter did not rank all candidates, he or she disapproved of those not ranked. Thus, when the fallback process descends to a level at which a voter no longer ranks candidates, that voter is assumed to approve of no additional candidates should the process continue to descend for other voters because no candidate has yet reached majority approval. Bucklin's system is FV absent the designation of approved candidates, who are implicitly assumed to be only those candidates that voters rank.

In the analysis of $\mathrm{FV}$ that follows, we assume that voters have preferences for all candidates, though they reveal their rankings only for approved candidates. As we will 
see, the non-revealed information may lead to the election of different candidates from PAV. First, however, we indicate properties that FV shares with PAV.

Proposition 5 Condorcet winners and unanimous $A V$ winners may not be $F V$ winners, whereas least-approved candidates may be FV winners.

Proof

In Example 1, there is no level 1 winner. Because candidate $b$ is the only candidate approved of by a majority (voters $I I$ and $I I I$ ) at level $2, b$ is the FV winner, whereas candidate $a$ is the Condorcet winner.

In Example 4, candidate $a$ is the FV winner at level 1, but candidate $b$ is the unanimous $\mathrm{AV}$ winner. In Example 6, candidate $a$ is the FV winner at level 1, but $a$ is the least approved of the four candidates. Q.E.D.

While FV and PAV share the properties listed in Proposition 5, FV, unlike PAV, may fail to elect a majority-preferred candidate among the majority-approved candidates.

Proposition 6 Suppose there are two or more majority-approved candidates. If one is majority-preferred among them, FV may not elect him or her.

Proof Consider the following 5-voter, 4-candidate example:

\section{Example 7}

- $I .2$ voters: $a b \mid c d$

- $I I .1$ voter: $d c a \mid b$

- III. 2 voters: $c a \mid b d$

There is no level 1 majority-approved candidate with at least 3 votes. Because candidate $a$ receives more approval (4 votes) than candidate $c$ ( 3 votes) at level 2, $a$ is the FV winner. But candidate $c$ is majority-preferred to candidate $a$ by 3 votes to 2. Q.E.D.

In fact, candidate $c$ is the Condorcet winner among all candidates, defeating candidates $b$ and $d$ as well. PAV, because of rule 2(i), picks candidate $c$, even though candidate $a$ is more approved at level 2 and is unanimously approved at level 3 (to which FV never descends).

A similar conflict between FV and PAV may occur when there is no Condorcet winner.

Proposition 7 A unanimously approved candidate in a cycle may not be the FV winner. 
Proof Consider the following 9-voter, 4-candidate example:

\section{Example 8}

- I. 2 voters: $a b c \mid d$

- II. 3 voters: $b d c \mid a$

- III. 4 voters: $c a \mid d b$

There is a cycle whereby $a>b>c>a$. candidate $c$ is the only candidate approved of by all 9 voters and so would be the PAV winner under rule 2(ii). Under FV, no candidate is majority-approved at level 1 , but at level 2 candidate $a$ receives 6 votes and candidate $b$ receives 5 votes, making $a$ the $\mathrm{FV}$ winner. Q.E.D.

Proposition $8 \mathrm{FV}, \mathrm{PAV}$, and AV may all give different winners for the same preferenceapproval profile.

Proof Consider the following 9-voter, 4-candidate example:

\section{Example 9}

- I. 4 voters: $a b c \mid d$

- II. 3 voters: $b c \mid a d$

- III. 2 voters: $d a c \mid b$

There is no level 1 majority-approved candidate, but candidates $a$ and $b$ each receive majority approval (6 and 7 votes, respectively) at level 2 . Because candidate $b$ (7 votes) is more approved of than candidate $a$ (6 votes), FV elects candidate $b$. But candidate $c$ is unanimously approved (9 votes) - at level 3 for the class $I$ and $I I I$ voters (to which FV never descends) - so AV elects candidate $c$. Finally, PAV elects candidate $a$, who is majority-preferred to the two other majority-approved candidates, $b$ and $c$. Q.E.D.

Note in Example 9 that no class of voters ranks the unanimously approved AV winner (candidate $c$ ) first, so he or she is likely to be only a lukewarm choice of everybody. Neither FV nor PAV favors such candidates if there are majority-approved candidates ranked higher by the voters.

In Examples 7, 8, and 9, one can determine from the rankings of the approved candidates that candidate $a$ is majority-preferred to candidate $b$. Thus in Example 9, even 
though the class $I I$ voters do not indicate that they prefer candidate $b$ to candidate $a$ when they rank their two approved candidates, $b$ and $c$, the fact these voters do not approve of candidate $a$ implies that candidate $b$, whom they do approve of, is ranked higher than candidate $a$. Similarly, one can ascertain from the ranking of the class $I I I$ voters that they prefer candidate $a$ to candidate $b$.

That PAV would have given a different outcome from FV may not always be revealed.

Proposition 9 Information used to determine an FV winner may not reveal that PAV would have chosen a different winner.

Proof Consider the following 3-voter, 4-candidate example:

\section{Example 10}

- $I .1$ voter: $a b c \mid d$

- II. 1 voter: $b d a \mid c$

- III. 1 voter: $c \mid a b d$

There is no level 1 majority-approved candidate, but at level 2 candidate $b$ receives majority approval (2 votes) and is, therefore, the $\mathrm{FV}$ winner. Because the class $I I I$ voter does not rank candidates below candidate $c$ under FV, it would not be known whether candidate $a$ would defeat candidate $b$, or vice versa, in a pairwise contest between these two candidates (while candidate $a$ is preferred by the class $I$ voter, candidate $b$ is preferred by the class $I I$ voter, leaving the contest undecided). But under PAV, wherein voters rank all candidates, the fact that the class $I I I$ voter prefers $a$ to $b$ would not only be revealed but also would render candidate $a$ the winner, because $a$ is majority-preferred to $b$. Q.E.D.

That FV ignores information on the lower-level preferences of voters is one reason why it gives different outcomes from PAV. Although we think information on nonapproved candidates should not be ignored, we recognize that it sometimes may be difficult for voters to provide it.

\section{Monotonicity of PAV and FV}

Such well-known voting systems as STV—also called "instant runoff voting" (IRV)—do not satisfy a property called "monotonicity," rendering them vulnerable to what Brams and Fishburn (2002, p. 215) call "ranking paradoxes." As an example of such a paradox, a voter may, by ranking a candidate first, cause him or her to lose, whereas this voter, by 
ranking the candidate last, enable him or her to win-just the opposite effect of what one would expect a top ranking to have.

Because PAV and FV are hybrid voting systems, it is useful to define two kinds of monotonicity.

1. A voting system is approval-monotonic if a class of voters, by approving of a new candidate-without changing their approval of other candidates-never hurts and may help this candidate get elected.

2. A voting system is rank-monotonic if a class of voters, by raising a candidate in their ranking — without changing their ranking of other candidates - never hurts and may help this candidate get elected.

A monotonicity paradox occurs when a voting system is not approval-monotonic or rank-monotonic; violations of rank-monotonicity have been investigated by Fishburn (1982), among others.

\section{Proposition $10 P A V$ and $F V$ are approval-monotonic.}

\section{Proof}

Consider PAV. Under rule 1, a class of voters, by approving of a candidate, helps him or her become the unique $\mathrm{AV}$, and therefore the PAV, winner. Under rule 2(i), a class of voters, by approving of a candidate, helps him or her become one of the majorityapproved candidates and, therefore, a possible PAV winner. Under rule 2(ii), a class of voters, by approving of a candidate, helps him or her become the AV, and therefore the PAV, winner among the majority-approved candidates in a cycle. Consider FV. Approving of a candidate allows him or her to be ranked and receive votes in the descent, thereby helping him or her become the FV winner. Q.E.D.

\section{Proposition $11 P A V$ and FV are rank-monotonic.}

\section{Proof}

Consider PAV. Under rule 1, ranks have no effect. Under rule 2(i), a class of voters, by raising a candidate in their ranking, helps that candidate defeat other majority-approved candidates in pairwise contests and thereby become the PAV winner. Under rule 2(ii), a class of voters, by raising a candidate in their ranking, helps that candidate be a member of the cycle-if there is no majority-preferred candidate among the majority-approved candidates - and thereby become a possible PAV winner. Consider FV. A class of voters, 
by raising a candidate in their ranking, helps that candidate become majority-approved at an earlier level, or receive the largest majority if two or more candidates are majorityapproved at the same level, and thereby become the FV winner. Q.E.D.

Thus, a class of voters can rest assured that giving either approval or a higher ranking to a candidate can never hurt and may help him or her get elected under PAV and FV. However, this may lead to the defeat of an already approved candidate that one prefers, which is illustrated by the following 7-voter, 4-candidate example:

\section{Example 11}

- $I .1$ voter: $a b \mid c d$

- II. 3 voters: $b \mid a c d$

- III. 2 voters: $c a \mid b d$

- $I V .1$ voter: $d \mid a b c$

Under PAV, candidate $b$ is the only candidate to be majority-approved (4 votes) and so is the PAV winner under rule 1.

But now assume that the 3 class $I I$ voters approve of candidate $a$ as well as candidate $b$ :

$I I^{\prime} .3$ voters: $b a \mid c d$

candidate $a$ receives 5 votes and candidate $b 4$ votes, so both are majority-approved. But because candidate $a$ is majority-preferred to candidate $b$ by 4 votes to 3 , candidate $a$ is the PAV winner under rule 2(i), contrary to the interests of the class $I I$ voters who switched from strategy $b$ to strategy $b a$.

Similarly, for the original approval strategies of the voters in Example 11, candidate $a$ is the FV winner, picking up 4 votes at level 2. But when the class $I I$ voters switch from strategy $b$ to strategy $b a$, candidate $a$ wins with 5 votes at level 2 . As under PAV, the strategy shift by the class $I I$ voters is detrimental to their interests

In section 7, we will show how information from polls may affect voters' calculations about how many candidates to approve of under PAV, and to approve of and rank under FV. As we will see, these calculations may or may not result in equilibrium outcomes.

The stability of outcomes under PAV and under FV reflects their robustness against manipulation. Stability may be looked at in either static or dynamic terms. In section 6 we view it statically - when will voters be motivated to try or not try to upset an outcome?whereas in section 7 we analyze how unstable outcomes, based on a dynamic poll model, evolve over time. 


\section{Nash Equilibria under PAV and FV}

Because PAV and FV give the same outcome as AV when either no candidate or one candidate receives the approval of a majority, they share many of the properties of AV. For example, in a field in which at most one candidate is likely to obtain majority approval, $\mathrm{PAV}$ and $\mathrm{FV}$, like $\mathrm{AV}$, give candidates an incentive to broaden their appeal to try to maximize their level of approval.

When candidates reach out to try to attract more votes, voters are likely to consider them acceptable and approve of more than one candidate. But if more than one candidate actually receives majority approval, the preferences of voters under PAV and FV matter, so the most-approved candidate may not win, as we showed earlier. Thus, a key question that both PAV and FV raise is how many candidates a voter should approve of if he or she deems more than one acceptable. As we showed in section 5, sometimes voting for additional candidates may sabotage the election of a preferred candidate.

In the analysis that follows, we assume that voters, in order to try to elect their preferred candidates, choose strategically where to draw the line between approved and disapproved candidates. But we assume that they are truthful in their rankings of candidates, which is equivalent to assuming that they choose from among their admissible and sincere AV strategies.

An AV strategy $\mathrm{S}$ is admissible if it is not dominated in a game-theoretic sensethat is, there is no other strategy that in all contingencies leads to at least as good an outcome and in some contingency a better outcome. Admissible strategies under AV involve always approving of a most-preferred candidate and never approving of a leastpreferred candidate (Brams and Fishburn, 1978, 1983).

An AV strategy $\mathrm{S}$ is sincere if, given the lowest-ranked candidate that a voter considers acceptable, he or she also approves of all candidates ranked higher. Thus, if $\mathrm{S}$ is sincere, there are no "holes" in a voter's approval set: Everybody ranked above a voter's lowest-ranked, but acceptable, candidate is also approved; and everybody ranked below this candidate is not approved.

As we will illustrate shortly, voters may have multiple sincere strategies, which some analysts consider desirable but which others consider problematic; this clash has sparked considerable controversy about AV. Given the multiplicity of sincere strategies, we are led to ask what, if any, strategies are stable under PAV and FV.

We define an outcome to be in equilibrium if the approval strategies of each preference class of voters that produce it constitute a Nash equilibrium. At such an equilibrium, no class of voters has an incentive to depart unilaterally from its approval strategy, because it would induce no better an outcome, and possibly a worse one, by doing so.

Proposition 12 Truth-telling strategies of voters under PAV and FV may not be in equi- 
librium. In particular, voters may induce a better outcome either by contracting or expanding their approval sets.

Proof We first prove this proposition for PAV using the following 7-voter, 4-candidate example:

\section{Example 12}

- $I .3$ voters: $a b \mid c d$

- II. 2 voters: $c \mid a b d$

- III. 2 voters: $d b \mid a c$

Candidate $b$, approved of by 5 voters, is the only candidate approved of by a majority and so is the PAV winner.

Now assume that the 3 class $I$ voters contract their approval set from strategy $a b$ to strategy $a$ :

$I^{\prime} .3$ voters: $a \mid b c d$

Then candidate $a$, who is preferred by the class $I$ voters to candidate $b$, will win under PAV rule 1 , receiving 3 votes to 2 votes each for candidates $b, c$, and $d$.

Next assume the 2 class $I I$ voters expand their approval set from strategy $c$ to strategy $c a$ :

$I I^{\prime} .2$ voters: $c a \mid b d$

Then candidates $a$ and $b$ tie with 5 votes each (candidates $c$ and $d$ receive 3 and 2 votes, respectively). Because candidates $a$ and $b$ both receive majority approval, we apply PAV rule 2(i). Since candidate $a$ is preferred to candidate $b$ by a majority of 5 votes to 2 , candidate $a$, whom the class $I I$ voters prefer to candidate $b$, is the winner.

Thereby both the contraction and the expansion of an approval set by a class of voters may induce a preferred outcome, rendering PAV strategies in Example 12 not in equilibrium. It is easy to show that the same contraction and expansion of approval sets induces preferred outcomes under FV (candidate $a$ instead of candidate $b$ in the case of contraction $I^{\prime}$; a tie between candidates $a$ and $b$ in the case of expansion $I I^{\prime}$ ). Q.E.D.

We showed earlier that PAV, FV, and $\mathrm{AV}$ may lead to three different outcomes for the same preference-approval profile (Proposition 8). The fact that an outcome is in equilibrium under one system, however, does not imply that it is in equilibrium under another system. 
Proposition 13 When PAV and FV give different outcomes, one may be in equilibrium and the other not.

\section{Proof}

In Example 9, we showed that candidate $a$ (the Condorcet winner) wins under PAV and candidate $b$ wins under FV. candidate $a$ is in equilibrium under PAV, because none of the three classes of voters, by switching to a different approval strategy, can induce an outcome they prefer to candidate $a$. On the other hand, candidate $b$ is not in equilibrium under FV, because the 4 class $I$ voters, by switching from strategy $a b c$ to $a$, can induce the election of candidate $a$, whom they prefer to candidate $b$. This example shows that PAV may give an equilibrium outcome when FV does not.

To show that FV may give an equilibrium outcome when PAV does not, consider the following example:

\section{Example 13}

- $I .1$ voter: $a b \mid c d$

- $I I .1$ voter: $c a \mid d b$

- III. 1 voter: $c \mid b$ ad

- $I V .1$ voter: $d b \mid a c$

- $V .1$ voter: $d b \mid c a$

Candidate $b$ is the only candidate approved of by a majority of 3 voters. No voter, by switching to a different approval strategy under FV, can induce a preferred outcome to candidate $b$ at level 2, making candidate $b$ an equilibrium outcome. candidate $b$, being the sole majority-approved candidate, is also the winner under PAV. But voter $I I$, by switching from strategy $c a$ to $c a d$, can render both candidates $d$ and $b$ majority-approved ( 3 votes each). Since $d$ is preferred to $b$ by a majority of 3 voters, including voter $I I$, voter II would have an incentive to induce this tied outcome under PAV, showing that FV may give an equilibrium outcome when PAV does not. Q.E.D.

The fact that equilibria under PAV do not imply equilibria under FV, or vice versa, indicates that one system is not inherently more stable than the other. 


\section{The Effects of Polls in 3-Candidate Elections}

In elections for major public office in the United States and other democracies, voters are not in the dark. Polls provide them with information about the relative standing of candidates and may also pinpoint their appeal, or lack thereof, to voters.

In this section, we focus on 3-candidate elections, because they are the simplest example in which information about the relative standing of candidates can affect the strategic choices of voters. Also, such elections are relatively common. We will show how voter responses to a sequence of polls may dynamically change outcomes under PAV and FV.

To assess the effects of polls in 3-candidate elections, we make the following assumptions:

1. No majority winner. None of the three candidates, $a, b$, or $c$, is the top choice of a majority of voters.

2. Initial support of only top choice. Before the poll, each voter approves of only his or her top choice.

3. Poll information. The poll indicates the relative standing of the candidates. For example, the ordering $n_{a}>n_{b}>n_{c}$ indicates that candidate $a$ receives the most approval votes, candidate $b$ the next most, and candidate $c$ the fewest (for simplicity, we do not allow for ties).

4. Strategy shifts. After the results of the poll are announced, voters may shift strategies by approving of a second choice as well as a top choice. Voters will vote for their two top choices if and only if the poll indicates (i) the about-to-become-winner is their worst choice and (ii) they can prevent this outcome by approving of a second choice, too, given they did not previously approve of this choice.

5. Repeated responses. After voters respond to a poll, they respond to new information that is revealed in subsequent polls, as described in assumption 4 above.

6. Termination. Voters cease their strategy shifts when they cannot induce a preferred outcome.

We assume that voters truthfully rank the three candidates at the start and do not change these rankings in response to the initial poll or any subsequent poll. We next investigate what outcomes occur in response to polls under PAV for two different kinds of preferences.

1. Single-peaked preferences: Voters perceive the candidates to be arrayed along a left-right continuum, with candidate $a$ on the left, candidate $b$ in the middle, and candidate 
$c$ on the right. Each voter most prefers one of these candidates, next most prefers an adjacent candidate, and least prefers the candidate farthest from his or her most-preferred candidate, who may or may not be adjacent.

More specifically, $a$-voters on the left with preference ranking $a b c$ may switch from strategy $a$ to strategy $a b$, whereas $c$-voters on the right with preference ranking $c b a$ may switch from strategy $c$ to strategy $c b$. The $b$-voters in the middle split into two groups, with one group preferring candidate $a$ over candidate $c(b a c)$ and the other group preferring candidate $c$ over candidate $a\left(\begin{array}{lll}b & c\end{array}\right)$. The former group may switch from strategy $b$ to strategy $b a$, whereas the latter group may switch from strategy $b$ to strategy $b c$.

Because preferences are single-peaked, the median candidate, $b$, is the unique Condorcet winner-he or she is preferred by a majority to both candidate $a$ and candidate c. We show in Table 1 three qualitatively different poll rankings that the initial poll may give:

(i) $n_{a}>n_{b}>n_{c}$; (ii) $n_{a}>n_{c}>n_{b}$; (iii) $n_{b}>n_{a}>n_{c}$,

\section{Table 1 about here}

where $n_{i}$ indicates the number of approval voters of candidate $i$. If the roles of candidates $a$ and $c$ are reversed, there are three analogous rankings, which we do not show in Table 1 :

(iv) $n_{c}>n_{b}>n_{a}$; (v) $n_{c}>n_{a}>n_{b}$; (vi) $n_{b}>n_{c}>n_{a}$.

For poll ranking (i) in Table 1, the voters with preference rankings $b c a$ and $c b a$ will switch from strategies $b$ and $c$, respectively, to strategies $b c$ and $c b$ to try to prevent their worst choice, candidate $a$, from winning (assumption 4). This results in the election of candidate $b$, whether candidate $b$ is the unique majority-approved candidate-with approval from three classes of voters-or candidate $c$ also wins a majority-with approval from two classes of voters-in which case candidate $b$ will defeat candidate $c$ in a pairwise contest. Because no voters can effect a preferred outcome under PAV through any subsequent shifts in their strategies-in response to a poll that shows candidate $b$ to be the unique or largest-majority winner-no voters will have an incentive to make further shifts.

The same shifts will occur for poll ranking (ii), again boosting candidate $b$ to winning status. As for poll ranking (iii), no voters will have an incentive to shift in response to the initial poll, because the plurality winner, candidate $b$, is not the worst choice of any voters.

Under FV, candidate $b$ will also prevail. In the case of poll rankings (i) and (ii), this occurs because candidate $b$ is the unique or largest-majority winner after the shift. In the 
case of poll ranking (iii), candidate $b$ is the initial plurality winner, after which the descent of voters ceases because no voter ranks $b$ last.

In summary, whichever of the three qualitatively different poll rankings occurs when voter preferences are single-peaked, the responses of voters to an initial poll leads to the election of Condorcet winner $b$ under both PAV and FV. But when preferences are cyclical and there is no Condorcet winner, the evolution of a winner is more drawn out, requiring up to three shifts rather than just one.

2. Cyclical preferences: We consider the simplest case of cyclical preferences, wherein three classes of voters, none with a majority of votes initially, have preferences $a$ $b c, b c a$, and $c a b$, so $a>b>c>a$. For simplicity, we exclude voters with preferences that do not contribute to the cyclic component of these voters (e.g., $a c b$ ).

If, as assumed earlier, voters initially approve of only their top choices, there are two qualitatively different poll rankings that the initial poll may give:

(i) $n_{a}>n_{b}>n_{c}$; (ii) $n_{a}>n_{c}>n_{b}$.

The four other possible rankings are analogous, with candidate $b$ ranked first in two cases and candidate $c$ ranked first in the other two:

(iii) $n_{b}>n_{a}>n_{c}$; (iv) $n_{b}>n_{c}>n_{a}$; (v) $n_{c}>n_{a}>n_{b}$; (vi) $n_{c}>n_{b}>n_{a}$.

In Table 2, we show the strategy shifts that voters will make in response to poll

\section{Table 2 about here}

rankings (i) and (ii). After an initial poll that shows candidate $a$ to be in first place in each case, there will be one shift by the $b c a$ voters (Shift $I$ ) —and up to two additional shifts (Shift $I I$ and Shift $I I I$ ) in response to subsequent polls that show other candidates to be in first place—as voters try to prevent their worst choice from winning.

To illustrate for poll ranking (i), the $b c a$ voters will switch from strategy $b$ to strategy $b c$ in Shift $I$ to try to prevent candidate $a$ from winning with a plurality of votes. But when this shift leads to candidate $c$ 's receiving a majority of votes, the $a b c$ voters will switch from strategy $a$ to strategy $a b$ in Shift $I I$, giving candidates $b$ and $c$ each a majority.

Under PAV, candidate $b$ will be majority-preferred to candidate $c$ in the contest between these two majority-approved candidates after Shift $I I$. Under FV, candidate $b$, with approval from both $a b c$ and $b c a$ voters at level 2, will receive a larger majority than candidate $c$ - based on the initial poll ranking — with approval from $b c a$ and $c a b$ voters.

At this stage, even if the $c a b$ voters switched from strategy $c$ to strategy $c a$, they could not induce the election of candidate $a$, who will get a smaller majority than candidate $b$, based on the initial poll ranking. Hence, the shifts will terminate after shift $I I$, resulting 
in the election of candidate $b$, the candidate with more first and second-place approval than any other candidate.

For poll ranking (ii), three shifts are required to induce the election of candidate $a$. In the absence of a Condorcet winner, the most approved candidate in the cycle-when all voters support their two top candidates-emerges as the winner under PAV and FV.

In summary, when preferences are cyclical, the candidate who is ranked first or second by the most voters prevails after three shifts under both PAV and FV. Together with our results on single-peaked preferences, we have the following:

Proposition 14 In the poll model for 3-candidate elections under PAV and FV, strategy shifts result in the election of (1) the Condorcet winner if preferences are singlepeaked and (2) the candidate ranked first or second by the most voters if preferences are cyclical.

These outcomes, however, may not be stable.

Proposition 15 In the poll model for 3-candidate elections under PAV and FV, strategy shifts may result in outcomes that are not in equilibrium when there is a Condorcet winner.

\section{Proof}

Assume that voter preferences are single-peaked (Table 1), and consider poll ranking (ii) after the shift. Assume that the $b c a$ and $c b a$ voters constitute a majority. Then the $c b a$ voters, by switching from strategy $c b$ to strategy $c$ (a contraction), will induce the election of candidate $c$, whom they prefer to candidate $b$. As the sole majority-approved candidate, candidate $c$ wins under both PAV and FV, rendering candidate $b$ not in equilibrium. Q.E.D.

Surprisingly, it is not the cyclical preferences of voters (in Table 2) that produce instability but the single-peaked preferences of voters (in Table 1) for poll ranking (ii) —and poll ranking (i) as well if the $b c a$ and $c b a$ voters constitute a majority in this situationthat produce instability. Thus, the strategy shifts of voters in response to polls, while leading to the outcomes indicated in Proposition 14, may not terminate at these outcomes because of the possible nonequilibrium status of candidate $b$ for poll rankings (i) and (ii) in Table 1.

This is not to say that the Condorcet winner (in Table 1), candidate $b$, cannot be supported as a Nash equilibrium in this situation. It turns out that the "critical strategy profile" of candidate $b$, 
$a b|c ; b| a c ; b|c a ; c b| a$, which maximizes $b$ 's approval vis-à-vis the other candidates, supports $b$ as a strong Nash equilibrium - no coalition of voter classes, by choosing different approval strategies, can induce an outcome they prefer to candidate $b$. Not only is it impossible for a coalition to replace $b$ with a preferred candidate under PAV and FV, but this is also true of $\mathrm{AV}$. In fact, under $\mathrm{AV}$, candidates are strong Nash equilibria at their critical strategy profiles if and only if they are Condorcet winners (Brams and Sanver, 2006).

We have assumed up until now that while voters may changes their levels of approval in order to try to induce preferred outcomes, they are steadfast in their rankings of candidates, which we assumed are truthful. But what if they can falsify their rankings? Then the candidates will be more vulnerable. But falsifying rankings, especially if information is incomplete, is a risky strategy that many voters are likely to shun.

\section{Conclusions}

It is worth emphasizing that PAV and FV duplicate $\mathrm{AV}$ when at most one candidate receives a majority of approval votes. In such a situation, there seems good reason to elect the AV winner, because if there is a different Condorcet winner, he or she would not be majority-approved. If the $\mathrm{AV}$ winner also is not majority-approved, his or her election seems even more compelling, because this is the most acceptable candidate in a field in which nobody is approved of by a majority.

When two or more candidates are majority-approved, PAV and FV may elect different winners from AV, the Borda count, STV, and each other. PAV chooses the majoritypreferred candidate, if there is one, among those who are majority-approved, whereas FV chooses the first candidate to receive a unique or largest majority in the descent.

If there is no majority-preferred candidate among the majority-approved candidates, PAV chooses the most approved candidate in the cycle. FV does the same if this candidate is in the first set of candidates to receive majority approval in the descent; if not, a majority-approved candidate with less approval—but received earlier-will be the FV winner. PAV and FV winners, if different from the AV winner, are likely to have more coherent majoritarian positions, not just be the lukewarm choices of most voters.

Candidates with coherent positions are more likely to run if they believe, without egregious pandering, that they can win. Consequently, PAV and FV may well encourage candidates to enter the fray who might otherwise be deterred because they are unwilling to sacrifice their fundamental tenets in order to win.

PAV and FV afford voters the opportunity to approve of lower-ranked candidates without necessarily helping them to win. Unlike AV, in which voting for a less-preferred candidate can cause the displacement of a more-preferred candidate, PAV and FV impede this 
event, though they do not rule it out entirely, by taking into account voter preferences, not just approval, albeit in different ways. Both systems are approval-monotonic and rankmonotonic, so approving of a candidate or ranking him or her higher never hurts, and may help, this candidate to get elected.

PAV is more information-demanding than FV, which asks voters to rank only their approved candidates. Without complete information on preference rankings, FV is less able to ensure the election of a majority-preferred-or the most approved if there is no majority-preferred-candidate among the majority-approved candidates.

PAV and FV may elect different candidates in equilibrium if voters contract or expand their approval sets; neither system is inherently more stable than the other. In the 3candidate dynamic poll model, Condorcet winners are elected after one shift when voter preferences are single-peaked - though not always in equilibrium-whereas candidates ranked first or second by the most voters are equilibrium choices after several shifts when voter preferences are cyclic.

By combining information on approval and preferences, PAV and FV may yield outcomes that neither kind of information, by itself, produces. Although PAV is more likely to lead to majority-preferred winners among the majority-approved, its greater information demands of voters may make FV a better practical choice. Such trade-offs require careful consideration, as do other ways of mixing approval and preferences to coax better social choices out of a voting system.

\section{References}

Brams, Steven J. (1982). "Strategic Information and Voting Behavior." Society 19, no. 6 (September/October): 4-11.

Brams, Steven J., and Peter C. Fishburn (1978). "Approval Voting." American Political Science Review 72, no. 3 (September): 831-857.

Brams, Steven J., and Peter C. Fishburn (1983). Approval Voting. Cambridge, MA: Birkhäuser Boston.

Brams, Steven J., and Peter C. Fishburn (2002). "Voting Procedures." In Kenneth Arrow, Amartya Sen, and Kotaro Suzumura (eds.), Handbook of Social Choice and Welfare, vol. 1. Amsterdam: Elsevier Science, pp. 175-236.

Brams, Steven J., and Peter C. Fishburn (2005). "Going from Theory to Practice: The Mixed Success of Approval Voting." Social Choice and Welfare 25, no. 2: 457-474.

Brams, Steven J., Peter C. Fishburn, and Samuel Merrill, III (1988). "The Responsiveness of Approval Voting: Comments on Saari and Van Newenhizen" and "Rejoinder to Saari and Van Newenhizen." Public Choice 59: 121-131, 149. 
Brams, Steven J., and M. Remzi Sanver (2006). "Critical Strategies under Approval Voting: Who Gets Ruled in and Ruled out." Electoral Studies 25, no. 2 (June): 287-305.

Fishburn, Peter C. (1982). "Monotonicity Paradoxes in the Theory of Elections." Discrete Applied Mathematics 4, no. 2 (April): 119-134.

Hoag, Clarence Gilbert, and George Hervey Hallet, Jr. (1926). Proportional Representation. New York: Macmillan.

Hurwicz, Leonid, and Murat R. Sertel (1999). "Designing Mechanisms, in Particular for Electoral Systems: The Majoritarian Compromise." In Murat R. Sertel (ed.), Economic Design and Behaviour. London: Macmillan.

Meirowitz, Adam (2004). "Polling Games and Information Revelation in the Downsian Framework." Games and Economic Behavior 51: 464-489.

Ossipoff, Mike, and Warren D. Smith (2005). "Survey of Voting Methods that Avoid Favorite-Betrayal." Preprint available at http://math.temple.edu/ wds/crv/FBCsurvey.html

Saari, Donald G., and Jill Van Newenhizen (1988). "The Problem of Indeterminacy in Approval, Multiple, and Truncated Voting Systems" and "Is Approval Voting an "Unmitigated Evil'?: A Response to Brams, Fishburn, and Merrill.” Public Choice 59: 101-120, 133-147.

Sertel, Murat, and M. Remzi Sanver (1999). "Designing Public Choice Mechanisms." In Imed Limam (ed.). Institutional Reform and Development in the MENA Region. Cairo, Egypt: Arab Planning Institute, pp. 129-148.

Sertel, Murat, and Bilge Yilmaz (1999). "The Majoritarian Compromise Is MajoritarianOptimal and Subgame-Perfect Implementable." Social Choice and Welfare 16, no. 4 (August): 615-627.

Taylor, Alan D. (2005). Social Choice and the Mathematics of Manipulation. New York: Cambridge University Press.

Weber, Robert J. (1995). “Approval Voting.” Journal of Economic Perspectives 9, no. 2 (Winter): 39-49. 


\section{Tables}

\begin{tabular}{|c|c|c|c|}
\hline Poll Ranking & (i) $n_{a}>n_{b}>n_{c}$ & (ii) $n_{a}>n_{c}>n_{b}$ & (iii) $n_{b}>n_{a}>n_{c}$ \\
\hline \multirow{4}{*}{ Initial Strategies } & $a \mid b c$ & $a \mid b c$ & $a \mid b c$ \\
& $b \mid a c$ & $b \mid a c$ & $b \mid a c$ \\
& $b \mid c a$ & $b \mid c a$ & $b \mid c a$ \\
& $c \mid b a$ & $c \mid b a$ & $c \mid b a$ \\
\hline Outcome & $a$ & $a$ & $b$ \\
\hline Shift in Strategies & $a \mid b c$ & $a \mid b c$ & \\
(if any) after Initial & $b \mid a c$ & $b \mid a c$ & \\
Poll & $b c \mid a$ & $b c \mid a$ & \\
\hline Outcome & $c b \mid a$ & $c b \mid a$ & \\
\hline
\end{tabular}

Table 1: Strategy Switches of Voters in Response to a Poll under PAV and FV: Single-Peaked Preferences with Three Poll Rankings (b Condorcet Winner) 


\begin{tabular}{|c|c|c|}
\hline Poll Ranking & (i) $n_{a}>n_{b}>n_{c}$ & (ii) $n_{a}>n_{c}>n_{b}$ \\
\hline \multirow{3}{*}{ Initial Strategies } & $a \mid b c$ & $a \mid b c$ \\
& $b \mid c a$ & $b \mid c a$ \\
& $c \mid a b$ & $c \mid a b$ \\
\hline Outcome & $a$ & $a$ \\
\hline \multirow{3}{*}{ Shift I (Initial Poll) } & $a \mid b c$ & $a \mid b c$ \\
& $c \mid a b$ & $b c \mid a$ \\
\hline Outcome & $c$ & $c \mid a b$ \\
\hline \multirow{2}{*}{ Shift II (2 ${ }^{\text {nd }}$ Poll) } & $a b \mid c$ & $c \mid a$ \\
\hline Outcome & $c \mid a b$ & $b c \mid a$ \\
\hline \multirow{2}{*}{ Shift III (3 $3^{\text {rd }}$ Poll) } & $b$ & $c \mid a b$ \\
\hline Outcome & & $b b \mid c$ \\
\hline
\end{tabular}

Table 2: Strategy Switches of Voters in Response to a Poll under PAV and FV: Cyclic Preferences with Two Poll Rankings 